\title{
RESULTADOS DE LA ENCUESTA DE OPINIÓN SOBRE INTENCIÓN DE VOTO EN LAS ELECCIONES MUNICIPALES Y PRESIDENCIALES: UNMSM
}

\author{
Olga Solano ${ }^{*}$, Félix Bartolo ${ }^{* *}, V_{\text {íctor García }}^{* * *}$, Orlando Giraldo ${ }^{* * *}$, César Paez ${ }^{* * * *}$ \& Jacinto \\ Mendoza ${ }^{* * * * *}$
}

Resumen: En el presente trabajo presentamos los resultados de la encuesta de opinión de intención de voto de los alumnos de la UNMSM en las elecciones municipales del 2010 y en las presidenciales del 2011. Utilizamos el muestreo aleatorio estratificado con afijación proporcional al tamaño (Scheffer y Mendenhall, 2007) y se consideraron en total veinte facultades de la UNMSM como estratos. Las encuestas se llevaron a cabo en tres etapas, durante los meses de julio, agosto y setiembre. En cada etapa se consideró a 500 participantes los cuales fueron seleccionados de cada una de las veinte facultades, siguiendo el esquema de muestreo estratificado con afijación proporcional. Para el cálculo del tamaño de muestra se consideró un límite para el error de estimación del $4.41 \%$, con un nivel de confianza del $95 \%$ y la información proporcionada por el Sistema Único de Matricula (SUM), de los alumnos matriculados el primer semestre del año académico 2010. ( Solano et al. 2011a).

Palabras clave: Intención de voto, Elecciones Municipales y Presidenciales, Muestreo Aleatorio Estratificado, Tamaño de muestra.

\section{RESULTS OF THE SURVEY OF OPINION IN THE INTENTION TO VOTE IN MUNICIPAL AND PRESIDENTIAL ELECTIONS TO STUDENTS OF THE UNMSM}

\begin{abstract}
In the present work, we present the results of the opinion poll of voting intentions of the students of San Marcos University in municipal elections of 2010 and presidential elections of 2011. We used stratified random sampling with allocation proportional to size (Scheffer y Mendenhall, 2007) and considered the following layers: Faculty of Administrative Sciences, Faculty of Biological Sciences, among others, in total twenty faculties of San Marcos University. The surveys were carried out in three stages, during the months of July, August and September. At each stage was considered to 500 participants who were selected from each of the twenty faculties, following the stratified sampling scheme with proportional allocation. o calculate the sample size was considered a limit to the estimation error of $4.41 \%$ with a confidence level of $95 \%$ and the information provided by the Unified Registration System (SUM) of students matriculated the first semester of 2010 academic year. (Solano et al. 2011a).

Key words: Voting intention, Municipal and Presidential Elections, Stratified Random Sampling, Size of sample.
\end{abstract}

\section{Introducción}

En el mes de octubre del año 2010 se llevó a cabo las elecciones municipales y en el año 2011 se realizaron las elecciones presidenciales en nuestro país. Debido a estos acontecimientos políticos en nuestro

\footnotetext{
${ }^{*}$ UNMSMS, Facultad de Ciencias Matemáticas, e-mail: osolanod@unsmsm.edu.pe

${ }^{* *}$ UNMSM, Facultad de Ciencias Matemáticas, e-mail: fbartolog@unmsm.edu.pe

${ }^{* * *}$ UNMSM, Facultad de Ciencias Matemáticas, e-mail: vgarciah@unmsm.edu.pe

${ }^{* * * *}$ UNMSM, Facultad de Ciencias Matemáticas, e-mail: ogiraldol@unmsm.edu.pe

${ }^{* * * * *}$ UNMSM, Facultad de Ciencias Matemáticas, e-mail: cpaezr@unmsm.edu.pe

${ }^{* * * * * *}$ UNMSM, Facultad de Ciencias Matemáticas, e-mail: jmendoza@unmsm.edu.pe
} 
país el equipo de investigación se motivo para realizar un estudio sobre una encuesta de opinión de la intención de voto en las elecciones municipales y presidenciales de los alumnos de nuestra universidad. El diseño muestral de este estudio ha sido publicado en la revista PESQUIMAT (Solano et al. 2011a). En el presente artículo pretendemos mostrar los principales resultados de dicha investigación.

\section{Metodología}

La encuesta de opinión sobre la intención de voto en las elecciones municipales y presidenciales se realizó en la Universidad Nacional Mayor de San Marcos en Perú. La encuesta piloto se realizó en el mes de junio del año 2010 con la finalidad de detectar los posibles errores en el instrumento de medición diseñado para el estudio. La aplicación del cuestionario final a los alumnos de pregrado de la UNMSM, el cual se realizó en tres etapas: la primera etapa del nueve al 23 de julio; para la segunda etapa del 23 al 28 de agosto y la tercera etapa del 24 de setiembre al primero de octubre, próximas a las elecciones municipales de octubre del 2010.

Utilizamos el muestreo aleatorio estratificado con afijación proporcional al tamaño (Scheffer y Mendenhall, 2007) y se consideraron en total veinte facultades de la UNMSM como estratos. Las encuestas se llevaron a cabo en tres etapas, durante los meses de julio, agosto y setiembre del año 2010. En cada etapa se consideró a 500 participantes los cuales fueron seleccionados de cada una de las veinte facultades; siguiendo el esquema de muestreo estratificado con afijación proporcional. Para el cálculo del tamaño de muestra se consideró un límite para el error de estimación del $4.41 \%$, con un nivel de confianza del $95 \%$ y la información proporcionada por el Sistema Único de Matricula (SUM), de los alumnos matriculados el primer semestre del año académico 2010. (Solano et al. 2011a). El cuestionario final quedo diseñado de la siguiente manera:

Parte I: Perfil del estudiante Sanmarquino: características de los estudiantes de pregrado como género, edad, EAP, facultad, ciclo de estudios y nivel socioeconómico.

Parte II: Intención de voto: recojo de opiniones del estudiante sobre intención de voto de las elecciones municipales y presidenciales sobre algunas características de los postulantes de los diferentes partidos políticos. Para realizar el análisis estadístico fue utilizado el programa SPSS ((Statistical Package off Social Sciences), versión 18.

\section{Resultados}

A continuación presentamos los resultados más relevantes del presente estudio. En el cuadro 1, presentamos la distribución de la muestra de alumnos de pregrado obtenida en el mes de julio del año 2010 según género.

Cuadro 1: Alumnos encuestados de Pregrado según género. UNMSM - julio 2010

\begin{tabular}{|c|c|c|}
\hline Género & Frecuencia & Porcentaje \\
\hline Femenino & 210 & 42 \\
\hline Masculino & 290 & 58 \\
\hline Total & 500 & 100 \\
\hline
\end{tabular}

El $58 \%$ de los alumnos encuestados son de género masculino, mientras que el $42 \%$ son de género femenino.

Cuadro 2: Alumnos encuestados de Pregrado según nivel socioeconómico. UNMSM- julio 2010 


\begin{tabular}{|c|c|c|}
\hline Nivel socioeconómico & Frecuencia & Porcentaje \\
\hline Alto & 11 & 2.2 \\
\hline Medio & 400 & 80.0 \\
\hline Bajo & 84 & 16.8 \\
\hline Muy bajo & 5 & 1.0 \\
\hline Total & 500 & 100 \\
\hline
\end{tabular}

El $80 \%$ de los encuestados son de nivel socioeconómico medio, mientras que el $16,8 \%$ pertenecen al nivel bajo, solo el 2,2\% pertenecen al nivel alto.

\section{Intención de voto en las elecciones municipales 2010}

A continuación se muestran los resultados de la intención de voto de las elecciones municipales durante el mes de setiembre.

Cuadro 3: Intención de voto de los alumnos, para la alcaldía de Lima según género. UNMSM-setiembre 2010

\begin{tabular}{|c|c|c|c|c|c|c|}
\hline \multirow{2}{*}{ Candidatos } & \multicolumn{2}{|c|}{$\mathrm{F}$} & \multicolumn{2}{c|}{ M } & \multicolumn{2}{c|}{ Total } \\
\cline { 2 - 6 } & Nro. & $\%$ & Nro. & $\%$ & & \\
\hline Lourdes Flores & 55 & 26.19 & 69 & 32.86 & 124 & 24.80 \\
\hline Susana Villaran & 119 & 56.67 & 146 & 69.52 & 265 & 53.00 \\
\hline Humberto Lay & 15 & 7.14 & 23 & 10.95 & 38 & 7.60 \\
\hline Gonzalo Alegría & 10 & 4.76 & 16 & 7.62 & 26 & 5.20 \\
\hline Luís Iberico & 3 & 1.43 & 4 & 1.90 & 7 & 1.40 \\
\hline Fernando Andrade & 3 & 1.43 & 17 & 8.10 & 20 & 4.00 \\
\hline Alex Gonzales & 2 & 0.95 & 1 & 0.48 & 3 & 0.60 \\
\hline Fernando Altuve & 2 & 0.95 & 4 & 1.90 & 6 & 1.20 \\
\hline Raúl Canelo & 1 & 0.48 & 2 & 0.95 & 3 & 0.60 \\
\hline No precisa & 0 & 0.00 & 8 & 3.81 & 8 & 1.60 \\
\hline Total & 210 & 100 & 290 & 100 & 500 & 100 \\
\hline
\end{tabular}

El $56.67 \%$ de los encuestados de género femenino, en el mes de setiembre, votarían para la alcaldía de Lima por Susana Villarán, mientras que el $69.52 \%$ de los encuestados de género masculino también lo harían por ella. El $26.19 \%$ del género femenino votaría por Lourdes Flores, mientras que el $32.86 \%$ del género masculino votarían por la candidata de Unidad nacional. El $53 \%$ del total de los encuestados votaría por Susana Villarán, el $24.80 \%$ por Lourdes Flores, el $7.60 \%$ lo haría por Humberto Lay, el $5.2 \%$ por Gonzalo Alegría. Solo el $0.60 \%$ votaría por Raúl Canelo y otro $0.60 \%$ por Alex Gonzales.

Cuadro 4: Opinión de los alumnos de Pregrado, sobre las frases siguientes, según género. UNMSM-setiembre 2010

\begin{tabular}{|c|c|c|c|c|}
\hline \multicolumn{1}{|c|}{ Frases } & $\mathrm{F}$ & $\mathrm{M}$ & Total & $\%$ \\
\cline { 2 - 3 } & $\%$ & $\%$ & & \\
\hline $\begin{array}{l}\text { Es preferible un alcalde que no } \\
\text { robe aunuqe haga pocas obras }\end{array}$ & 15.71 & 18.62 & 87 & 17.40 \\
\hline $\begin{array}{c}\text { Es preferible un alcalde que haga } \\
\text { buenas obras aunque robe }\end{array}$ & 33.81 & 31.38 & 162 & 32.40 \\
\hline Ninguna de las anteriores & 50.00 & 50.00 & 250 & 50.00 \\
\hline No precisa Total & 0.48 & 0 & 1 & 0.20 \\
\hline & 100 & 100 & 500 & 100 \\
\hline
\end{tabular}

El $32.40 \%$ de los encuestados prefiere un alcalde que haga buenas obras aunque robe, mientras que el $17.40 \%$ prefiere a un alcalde que no robe aunque haga pocas obras, el $50 \%$ no prefiere ninguna de las dos frases. 
Cuadro 5: Opinión de los alumnos de Pregrado, sobre las frases siguientes, según intención de voto. UNMSM-setiembre 2010

\begin{tabular}{|c|c|c|c|c|}
\hline Frases & $\begin{array}{c}\text { Lourdes } \\
\text { Flores }\end{array}$ & $\begin{array}{c}\text { Susana } \\
\text { Villarán }\end{array}$ & $\begin{array}{c}\text { Hum. } \\
\text { Lay }\end{array}$ & $\begin{array}{c}\text { Fernando } \\
\text { Andrade }\end{array}$ \\
\hline $\begin{array}{c}\text { Es preferible un alcalde que no } \\
\text { robe aunque haga pocas obras }\end{array}$ & 17.74 & 17.36 & 21.05 & 20.00 \\
\hline $\begin{array}{c}\text { Es preferible un alcalde que haga } \\
\text { buenas obras aunque robe }\end{array}$ & 41.13 & 30.19 & 34.21 & 25.00 \\
\hline Ninguna de las anteriores & 41.13 & 52.08 & 44.74 & 55.00 \\
\hline No precisa Total & 0 & 0.38 & 0 & 0 \\
\hline & 100 & 100 & 100 & 100 \\
\hline
\end{tabular}

De los encuestados que prefieren a Lourdes Flores como alcaldesa, el $41.13 \%$ de ellos prefiere un alcalde que haga buenas obras aunque robe, el $17.74 \%$ prefiere un alcalde que no robe aunque haga pocas obras, el $41.13 \%$ no prefiere ninguna de las dos frases.

Cuadro 6: Opinión de los alumnos de Pregrado, independientemente de sus preferencias, políticas, respecto al candidato más capacitado(a) para...UNMSM-setiembre 2010

\begin{tabular}{|c|c|c|c|c|}
\hline Respuestas & Andrade & Flores & Lay & Villarán \\
\hline Combatir la corrupción & 12.9 & 18.60 & 23.47 & 42.49 \\
\hline $\begin{array}{l}\text { Mejorar la seguridad } \\
\text { ciudadana de Lima }\end{array}$ & 13.53 & 31.65 & 10.32 & 40.60 \\
\hline $\begin{array}{l}\text { Mejorar el sistema de } \\
\text { transporte }\end{array}$ & 14.47 & 36.72 & 7.13 & 35.85 \\
\hline $\begin{array}{l}\text { Mejorar el sistema } \\
\text { de recojo de basura }\end{array}$ & 18.09 & 24.94 & 10.27 & 41.08 \\
\hline $\begin{array}{l}\text { Prevenir y controlar la } \\
\text { contaminación ambiental }\end{array}$ & 10.53 & 20.57 & 13.64 & 49.28 \\
\hline $\begin{array}{l}\text { Gestionar el monto de los } \\
\text { arbitrios e impuestos }\end{array}$ & 12.78 & 25.31 & 13.02 & 43.00 \\
\hline $\begin{array}{l}\text { Realizar el mantenimiento } \\
\text { de calles y pistas }\end{array}$ & 14.43 & 30.93 & 10.31 & 36.86 \\
\hline
\end{tabular}

Hay un mayor porcentaje de encuestados que opina que Susana Villarán está más capacitada para: combatir la corrupción, mejorar la seguridad ciudadana de Lima, mejorar el sistema de recojo de basura, prevenir y controlar la contaminación ambiental, gestionar el monto de los arbitrios e impuestos municipales y realizar el mantenimiento de calles y pistas.El $36.72 \%$ de respuestas de los encuestados sobre mejorar el sistema de transporte opina que Lourdes está más capacitada para mejorar este sistema.

Cuadro 7: Opinión de los alumnos de Pregrado, independientemente de sus preferencias políticas, respecto a las cualidades que caracterizan mejor a cada candidato. UNMSM-setiembre 2010 


\begin{tabular}{|l|c|c|c|c|}
\hline \multicolumn{1}{|c|}{ Cualidades } & Andrade & Flores & Lay & Villarán \\
\hline Tiene objetivos y metas claras & 52 & 104 & 28 & 163 \\
\hline Ser un buen líder & 52 & 114 & 44 & 155 \\
\hline Experiencia en política & 77 & 195 & 21 & 83 \\
\hline $\begin{array}{l}\text { Capacidad para tomar } \\
\text { decisiones difíciles }\end{array}$ & 39 & 64 & 31 & 119 \\
\hline Honestidad y confiabilidad & 34 & 36 & 163 & 129 \\
\hline Firmeza y autoridad & 27 & 89 & 37 & 120 \\
\hline Preocuparse por la gente & 31 & 44 & 83 & 161 \\
\hline $\begin{array}{l}\text { Capacidad para convocar } \\
\text { a buenos técnicos }\end{array}$ & 45 & 63 & 31 & 102 \\
\hline $\begin{array}{l}\text { Experiencia en gestión y } \\
\text { administración }\end{array}$ & 56 & 78 & 22 & 85 \\
\hline
\end{tabular}

La mayoría de las respuestas de los encuestados sobre las cualidades de los candidatos opinan que Susana Villarán tiene objetivos y metas claras, es una buena líder, tiene capacidad para tomar decisiones difíciles, tiene firmeza y autoridad, se preocupa por la gente, tiene capacidad para convocar a buenos técnicos y tienen experiencia en gestión y administración; mientras que Lourdes Flores tiene experiencia en política y Humberto Lay es honesto y confiable.

Cuadro 8: Opinión de los alumnos de Pregrado, independientemente de sus preferencias políticas, respecto al candidato que considera usted que ... UNMSM - setiembre 2010

\begin{tabular}{|c|c|c|c|c|}
\hline \multicolumn{1}{|c|}{ Respuestas } & Flores & Villarán & Ambas & Ninguna \\
\hline $\begin{array}{c}\text { Está más asociada a la } \\
\text { lucha contra la corrupción }\end{array}$ & 22 & 48 & 15 & 15 \\
\hline $\begin{array}{l}\text { Ha tenido una mayor oposición } \\
\text { al acual gobierno }\end{array}$ & 17 & 49 & 11 & 24 \\
\hline Más experiencia en política & 56 & 21 & 16 & 7 \\
\hline $\begin{array}{l}\text { Mayor vínculación con el } \\
\text { actual gobierno }\end{array}$ & 58 & 16 & 10 & 16 \\
\hline Mayor capacidad de gestión & 29 & 42 & 19 & 10 \\
\hline Representa la política tradicional & 59 & 17 & 13 & 11 \\
\hline $\begin{array}{c}\text { Más vínculos con el mundo } \\
\text { empresarial }\end{array}$ & 57 & 18 & 15 & 10 \\
\hline $\begin{array}{c}\text { Continuará de mejor manera } \\
\text { la gestión del actual alcalde }\end{array}$ & 35 & 38 & 13 & 15 \\
\hline $\begin{array}{c}\text { Más interesada en las necesidades } \\
\text { de la mayoría de los ciudadanos }\end{array}$ & 16 & 52 & 15 & 18 \\
\hline
\end{tabular}

Hay un mayor porcentaje de encuestados que opina que la candidata a la alcaldía de Lima Susana Villarán, está más asociada a la lucha contra la corrupción, ha tenido una mayor oposición al actual gobierno, tiene mayor capacidad de gestión, continuará de mejor manera la gestión del actual alcalde y está más interesada en las necesidades de la mayoría de los ciudadanos de Lima; mientras que Lourdes Flores, tiene más experiencia en política, ha tenido mayor vinculación con el actual gobierno, representa la política tradicional y tienen más vínculos con el mundo empresarial.

Cuadro 9: Opinión de los alumnos de Pregrado, respecto a si aprueba o desaprueba el desempeño público de cada candidato. UNMSM-setiembre 2010 


\begin{tabular}{|l|c|c|c|}
\hline \multirow{2}{*}{ Candidatos } & ¿Aprueba el desem & peño público de...? & \multirow{2}{*}{ Total } \\
\cline { 2 - 3 } & $\mathrm{Si}$ & No & \\
\hline Lourdes Flores & 38.6 & 61.4 & 100 \\
\hline Susana Villarán & 67.4 & 32.6 & 100 \\
\hline Humberto Lay & 32.0 & 68.0 & 100 \\
\hline Gonzalo Alegría & 14.0 & 86.0 & 100 \\
\hline Luís Iberico & 12.0 & 88.0 & 100 \\
\hline Fernando Andrade & 27.0 & 73.0 & 100 \\
\hline Alex Gonzales & 6.8 & 93.2 & 100 \\
\hline Fernando Altuve & 6.0 & 94.0 & 100 \\
\hline Raúl Canelo & 4.4 & 95.6 & 100 \\
\hline
\end{tabular}

El $61.4 \%$ de los encuestados no aprueba el desempeño público de Lourdes Flores, mientras que el $67.4 \%$ aprueba el desempeño público de Susana Villarán. El $32 \%$ de los encuestados aprueba el desempeño público de Humberto Lay.

A continuación se muestran los resultados de la intención de voto de las elecciones municipales durante los meses de julio, agosto y setiembre del año 2010.

Cuadro 10: Intención de Voto de los alumnos de Pregrado según los meses de aplicación de la Encuesta (julio, agosto,setiembre). UNMSM-2010

\begin{tabular}{|c|c|c|c|}
\hline \multirow{2}{*}{ Candidatos } & \multicolumn{3}{|c|}{ Meses } \\
\cline { 2 - 4 } & Julio & Agosto & Setiembre \\
\cline { 2 - 4 } & $\%$ & $\%$ & $\%$ \\
\hline Lourdes Flores & 50.0 & 26.6 & 24.8 \\
\hline Alexander Kouri & 23.6 & 8.0 & \\
\hline Susana Villarán & 13.6 & 52.0 & 53.0 \\
\hline Humberto Lay & & 5.2 & 7.6 \\
\hline Gonzalo Alegría & & 1.4 & 5.2 \\
\hline Otros & 12.8 & 6.8 & 9.4 \\
\hline Total & 100 & 100 & 100 \\
\hline
\end{tabular}

En el Cuadro 10, observamos los resultados de la intención de voto de los alumnos de la UNMSM en las elecciones municipales durante los meses de julio hasta el mes de setiembre del año 2010, así tenemos que en la encuesta del mes de julio, la postulante del partido político del PPC (Partido Popular Cristiano) obtuvo el $50 \%$, mientras que el postulante del partido político de Cambio Radical obtuvo el $23.6 \%$, la postulante del partido político de Fuerza Social obtuvo el $13.6 \%$. En la encuesta del mes de agosto, la postulante del partido político de Fuerza Social obtuvo el $52 \%$ (incrementándose en $38.4 \%$ con respecto al mes anterior), mientras que la postulante del partido político del PPC-Unidad Nacional obtuvo el $26.6 \%$ (disminuyendo en $23.4 \%$ con respecto al mes anterior), el postulante del partido de Cambio Radical obtuvo el $8 \%$, mientras que el postulante del partido de Restauración Nacional obtuvo el $5.2 \%$ y el postulante del partido Acción Popular obtuvo el 1.4\%. Resultados próximos a las elecciones municipales, en la encuesta del mes de setiembre, la postulante del partido político de Fuerza Social obtuvo el 53\%, la postulante del partido político del PPC (Partido Popular Cristiano) - Unidad Nacional obtuvo el $24.8 \%$, mientras que el postulante del partido de Restauración Nacional obtuvo el $7.6 \%$, y el postulante del partido de Acción Popular obtuvo el $5.2 \%$. 
A continuación se muestran los resultados de la intención de voto de las elecciones presidenciales de los alumnos pregrado de la UNMSM entrevistados durante los meses de julio, agosto y setiembre.

Cuadro 11: Intención de Voto de los alumnos de Pregrado en las elecciones presidenciales según los meses de aplicación de la encuesta(julio, agosto,setiembre) UNMSM-2010

\begin{tabular}{|c|c|c|c|}
\hline \multirow{2}{*}{ Candidatos } & \multicolumn{3}{|c|}{ Meses } \\
\cline { 2 - 4 } & Julio & Agosto & Setiembre \\
\cline { 2 - 4 } & $\%$ & $\%$ & $\%$ \\
\hline Luís Castañeda & 38.2 & 34.4 & 31.6 \\
\hline Alejandro Toledo & 21.4 & 28.6 & 24.8 \\
\hline Keiko Fujimori & 15.4 & 25.0 & 24.6 \\
\hline Ollanta Humala & 5.0 & 4.2 & 6.2 \\
\hline Jayme Bayly & 4.6 & 4.2 & 6.2 \\
\hline Otros & 15.4 & 3.6 & 6.6 \\
\hline Total & 100 & 100 & 100 \\
\hline
\end{tabular}

En el Cuadro 11, observamos los resultados de la intención de voto de los alumnos de la UNMSM en las elecciones presidenciales durante los meses de julio hasta el mes de setiembre del año 2010, así tenemos que en la encuesta del mes de julio, el postulante del partido político de Solidaridad Nacional (psn), Luís Castañeda obtuvo el $38.2 \%$, mientras que el postulante del partido político Perú Posible obtuvo el $21.4 \%$, el postulante del partido político cambio 90 obtuvo el $15.4 \%$ y el postulante del partido Nacionalista Peruano obtuvo el 5\%, mientras que Jayme Bayly obtuvo el $4.6 \%$. En la encuesta del mes de agosto, el postulante del partido político de Solidaridad Nacional (psn), Luís Castañeda obtuvo el $34.4 \%$ (descendiendo en $3.8 \%$ con respecto al mes de julio), mientras que el postulante del partido político Perú Posible obtuvo el $28.6 \%$ (incrementándose $7.2 \%$ con respecto al mes anterior), el postulante del partido político cambio 90 obtuvo el $25 \%$ (incrementándose en $9.6 \%$ con respecto al mes anterior) y el postulante del partido Nacionalista Peruano obtuvo el $4.2 \%$, mientras que Jayme Bayly obtuvo el $4.2 \%$. En la encuesta del mes de setiembre, el postulante del partido político del Solidaridad Nacional (psn), Luís Castañeda obtuvo el $31.6 \%$ (descendiendo en $2.8 \%$ con respecto al mes de agosto), mientras que el postulante del partido político Perú Posible obtuvo el $24.8 \%$ (disminuyendo en 3,8\% con respecto al mes de agosto), la postulante del partido político cambio 90 obtuvo el $24.6 \%$ (disminuyendo levemente en $0.4 \%$ con respecto al mes de agosto) y el postulante del partido Nacionalista Peruano obtuvo el $6.2 \%$ incrementando en $2 \%$ con respecto al mes de agosto), mientras que Jayme Bayly obtuvo el $6.2 \%$ (incrementándose en $2 \%$ con respecto al mes anterior).

\section{Conclusiones}

Con respecto a las elecciones municipales, comparando los resultados de la presente investigación obtenidas en el mes de setiembre próximas a las elecciones municipales, con los resultados finales obtenidos por la ONPE, observamos que los resultados son similares. Nuestro estudio fue realizado en la UNMSM con una muestra de 500 alumnos, así tenemos que los resultados finales dados por la ONPE fueron: el $38.4 \%$ lo obtuvo la candidata del partido de Fuerza Social, el $37.6 \%$ lo obtuvo la candidata del PPCUnidad Nacional, el $8.6 \%$ lo obtuvo el candidato de Restauración Nacional, y el $4.2 \%$ lo obtuvo el candidato del Partido Democrático Somos Perú, mientras que en nuestro estudio en la encuesta realizada el mes de setiembre próximas a las elecciones municipales nuestros resultados fueron: el $53 \%$ para la candidata de Fuerza Social, el $24.8 \%$ para la candidata del PPC-Unidad Nacional, el $7.6 \%$ el candidato de Restauración Nacional y el $5.2 \%$ para el candidato del Partido Democrático Somos Perú.

Con respecto a la intención de voto de los alumnos de la UNMSM en las elecciones presidenciales durante los meses de julio hasta el mes de setiembre del año 2010, tenemos los resultados más resaltantes, en la encuesta realizada el mes de julio el postulante del partido político de Solidaridad Nacional Luís Castañeda obtuvo el $38.2 \%$, en el mes de agosto disminuye esta cifra en $3.9 \%$ con respecto al mes de 
julio, y en el mes de setiembre descendió en $2.8 \%$ respecto al mes de agosto. El postulante del partido político Perú Posible obtuvo el $21.4 \%$ en el mes de julio, en el mes de agosto esta cifra se incrementó en $7.2 \%$ con respecto al mes anterior y en el mes de setiembre disminuye esta cifra en $3.8 \%$ con respecto al mes de agosto. El postulante del partido político cambio 90 obtuvo el $15.4 \%$ en el mes de julio, en el mes de agosto esta cifra se incrementó en $9.6 \%$ con respecto al mes anterior y en el mes de setiembre disminuye en $0.4 \%$ con respecto al mes de agosto.

De los resultados se concluye que la Técnica del Muestreo Estratificado (Scheffer y Mendenhall, 2007; Cochran, 1977) fue útil y provechosa en una investigación de la realidad de nuestra sociedad, intención de voto de las elecciones municipales y presidenciales de los alumnos de la UNMSM, se recomienda seguir aplicando la técnica del muestreo en investigaciones de nuestra realidad.

\section{Agradecimiento}

Los autores expresamos nuestro agradecimiento al Consejo Superior de Investigaciones de la Universidad Nacional Mayor de San Marcos por el apoyo financiero para la ejecución del estudio motivo de la presente publicación. 


\section{Bibliografía}

[1] Aaker, A., Kumar, V., Day, G. (2007). Investigación de mercados. 4ta. Ed., Edit. Limusa, México.

[2] Cochran, G.W. (1977). Técnicas de muestreo. 5ta. Ed., Edit. Continental,. México.

[3] Des, R. (1980). Teoría del muestreo. 1ra. Ed., Edit. Fondo de cultura económica, México.

[4] Lohr, S. (2000). Muestreo: diseño y análisis. 2da. Ed., Edit. Thomson Editores, México.

[5] Malhotra, N. (1997). Investigación de mercados. 2da. Ed., Edit. Prentice Hall, México.

[6] Perez, C. (2006). Muestreo estadístico. 3ra. Ed., Edit. Pearson Educación, España.

[7] Scheaffer, R.; Mendenhall, W. Y; Ott, L. (2007). Elementos de muestreo. 6ta. Ed., Edit. Iberoamérica, México. 